\title{
Use of Phased Array Coils for a Determination of Absolute Metabolite Concentrations
}

\author{
Oliver Natt, Vitaliy Bezkorovaynyy, Thomas Michaelis, and Jens Frahm*
}

\begin{abstract}
This work describes the use of phased array coils for a quantification of absolute metabolite concentrations. The method is demonstrated for single-voxel localized proton MRS of human brain with an eight-element receive-only head coil. It is based on the transmitter reference amplitude of the body coil used for RF transmission. A relative sensitivity of every element of the phased array coil is derived from a combination of two reference scans without water suppression that correspond to either the body coil in transmit-receive mode or the phased array coil in conjunction with body coil excitation. Experimental results were obtained at $2.9 \mathrm{~T}$ for both phantoms and 12 human subjects in different locations of gray and white matter. The data demonstrate that the procedure is technically robust and without a penalty in measuring time. Moreover, it takes full advantage of the signal-to-noise gain for quantitative proton MRS and may be extended to other phased array coils without the need for a recalibration. Magn Reson Med 53: 3-8, 2005. @ 2004 Wiley-Liss, Inc.
\end{abstract}

Key words: magnetic resonance spectroscopy (MRS); phased array coils; cerebral metabolites; quantification; human brain

Because phased array coils offer improved signal-to-noise ratio (SNR) or speed (in conjunction with parallel acquisition techniques), most modern MRI systems are equipped with multiple-element arrays and a corresponding number of independent radiofrequency (RF) receiver channels. For localized MRS the expected SNR gain of phased array coils results from two sources. First, for volume-of-interest (VOI) locations close to a coil element, the higher sensitivity of a surface coil in comparison with a volume coil yields significant advantages, and second, the uncorrelated noise from different coil elements allows for a further improvement by a suitable combination of individual signals. Although the underlying phenomena of noise correlation and signal combination have previously been studied both theoretically (1) and experimentally (2-4), no attempt has been made to utilize receive-only phased array coils for a quantification of absolute metabolite concentrations.

The most simple methods for absolute quantification rely on either an internal reference such as the tissue water content $(5,6)$ or an external reference in the form of a model solution attached to the coil (7). Unfortunately, these approaches are often hampered by problems such as unknown (pathologic) changes in tissue water or the need for large off-center positions for the external reference phantom. The

Biomedizinische NMR Forschungs $\mathrm{GmbH}$ am Max-Planck-Institut für biophysikalische Chemie, Göttingen, Germany.

${ }^{*}$ Correspondence to: J. Frahm, Biomedizinische NMR Forschungs $\mathrm{GmbH}$, 37070 Göttingen, Germany. E-mail: jfrahm@gwdg.de

Received 12 July 2004; revised 25 August 2004; accepted 30 August 2004.

DOI 10.1002/mrm.20337

Published online in Wiley InterScience (www.interscience.wiley.com).

(c) 2004 Wiley-Liss, Inc. best results in terms of reproducibility and practicality have therefore been obtained with the use of the absolute reference method, which scales the acquired data by the amplitude of a nonselective $90^{\circ}$ reference pulse (8). The approach accounts for different coil loadings according to the reciprocity theorem $(9,10)$. Alternatively, the MRS signal may be scaled by the amplitude of a modified water suppression pulse $(11,12)$. Whereas both the internal and the external reference method require no adjustment for phased array coils, the absolute reference method cannot be directly applied to a situation where the excitation by a body coil is followed by signal detection with a receive-only coil. This is because the only available transmitter amplitude does not reflect the loading of the phased array coil but that of the body coil.

The purpose of this study was (i) to develop a method for the absolute quantification of metabolite concentrations with phased array coils and (ii) to prove its reliability by applications to single-voxel proton MRS of the human brain in vivo.

\section{MATERIALS AND METHODS}

All studies were conducted at 2.9 T (Siemens Magnetom Trio, Erlangen, Germany) using an eight-channel receiveonly phased array head coil (Siemens, Erlangen, Germany) in combination with a body coil, which can be used both in transmit-only and in transmit-receive modes.

Localized proton MRS of human brain was performed using a STEAM sequence (TR/TM/TE $=6000 / 10 / 20 \mathrm{msec}$, 2048 complex data points, dwell time $0.5 \mathrm{msec}$ ). For phantom studies the repetition time was prolonged to $20000 \mathrm{msec}$ to account for the longer $T_{1}$ relaxation times. Water suppression was accomplished with a preceding CHESS sequence (pulse spacing $60 \mathrm{msec}$ ) and flip angles of $89.2,83.4$, and $160.2^{\circ}$, respectively (13). All spectral analyses were performed with use of LCModel (14).

Localized MRS measurements consisted of three individual acquisitions: (i) a single scan without water suppression using the body coil in transmit-receive mode, (ii) a single scan without water suppression using the body coil for RF transmission and the phased array coil for signal detection, and (iii) the actual water-suppressed scan (64 accumulations for in vivo studies) using the same arrangement as in (ii). The contributions from the different coil elements were saved as independent data and therefore resulted in a set of 17 signals for one spectrum.

Before each examination, the body coil was automatically tuned and matched and a transmitter reference amplitude was determined by the manufacturer's software (NUMARIS/4, Siemens, Erlangen, Germany). The accuracy of this procedure was verified for both phantoms and human subjects by measuring the signal amplitude of lo- 


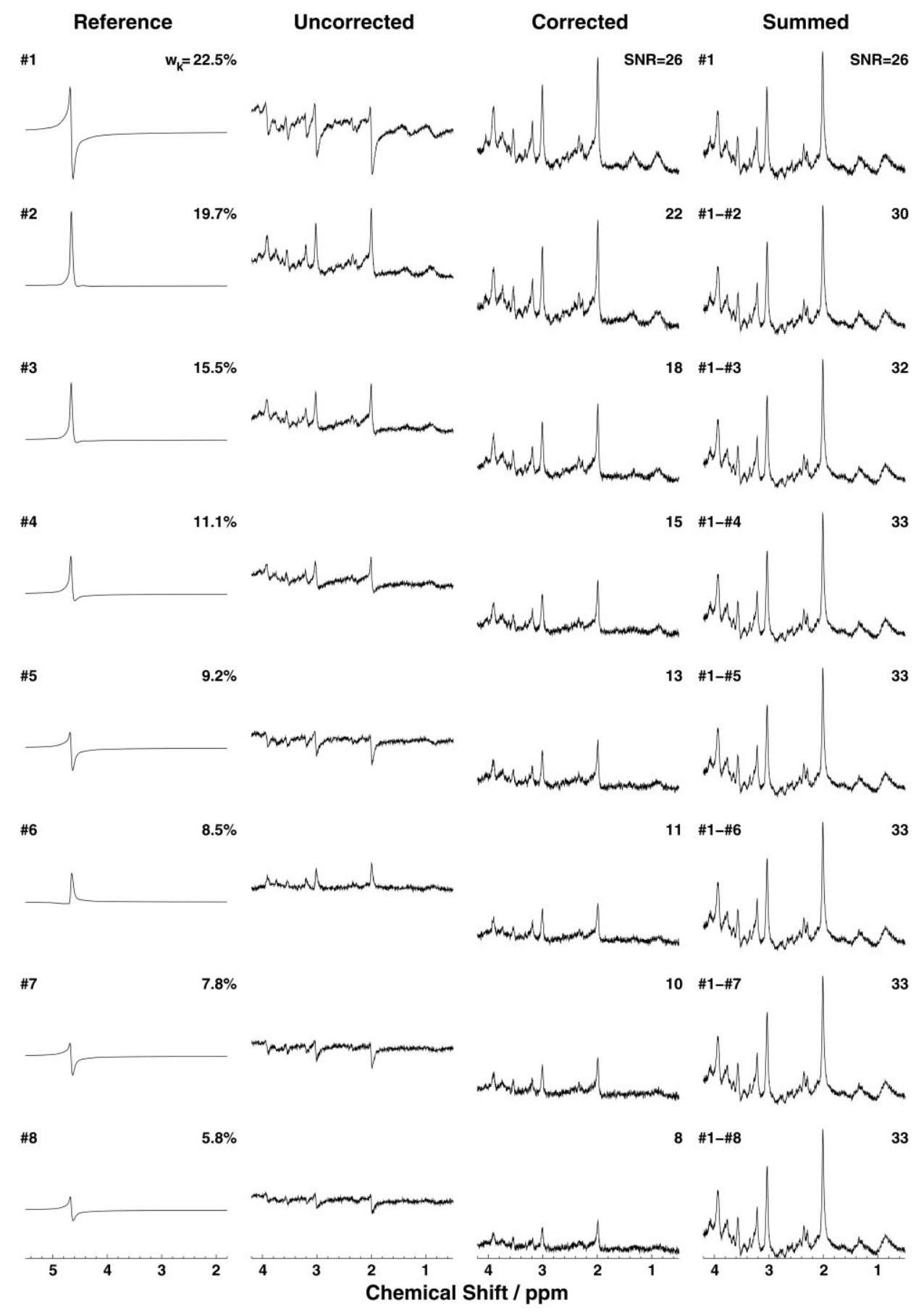

calized acquisitions without water suppression as a function of the (nominal) flip angle, that is at $\pm 5, \pm 10, \pm 15$, and $\pm 20 \%$ of the RF power calculated for $90^{\circ}$.

\section{Phantom Experiments}

Because the library of model spectra for the MRS analysis with LCModel was originally acquired with a volume coil, a calibration factor for the phased array coil was obtained by measuring a spherical phantom of $50 \mathrm{mM}$ creatine (Fluka 27890) in an aqueous phosphate buffer (72 $\mathrm{mM} \mathrm{K}_{2} \mathrm{HPO}_{4}$, $28 \mathrm{mM} \mathrm{KH}_{2} \mathrm{PO}_{4}$ ). During these calibration experiments various "load phantoms" were placed in the magnet bore in different positions in order to mimic the conditions encountered in vivo and to test the reproduciblity of the quantification procedure under different load conditions. The spatial homogeneity of the quantification procedure was examined using a large cylindrical ethanol phantom (200 mM ethanol in aqueous phosphate buffer as above) and multiple VOI positions $\left(20 \times 20 \times 20 \mathrm{~mm}^{3}\right)$ shifted along each axis until they were touching the borders of the phantom.

\section{Human Studies}

Twelve healthy volunteers (7 female/5 male, age range 21-38 years, mean $26.9 \pm 4.6$ years) participated in the 
FIG. 2. Deviation of the quantified ethanol concentration from the mean value as a function of VOI $\left(20 \times 20 \times 20 \mathrm{~mm}^{3}\right)$ position in left-right $(\mathrm{L}-\mathrm{R})$, anterior-posterior $(\mathrm{A}-\mathrm{P})$, and head-foot $(\mathrm{H}-\mathrm{F}) \mathrm{di}-$ rection.

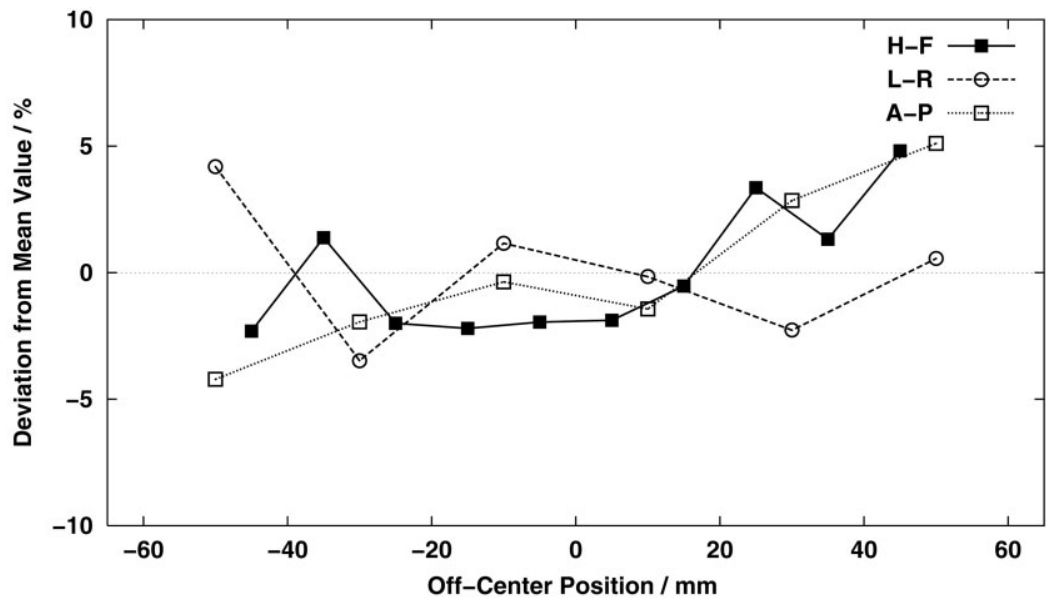

study. Informed written consent was obtained before all examinations. Each session comprised $T_{1}$-weighted MRI (RF spoiled 3D FLASH, TR/TE $=11 / 4.9 \mathrm{msec}$, flip angle $\left.15^{\circ}\right)$ in sagittal $\left(1 \times 1 \times 4 \mathrm{~mm}^{3}\right)$, coronal $\left(1 \times 1 \times 4 \mathrm{~mm}^{3}\right)$, and transverse orientation $\left(1 \times 1 \times 2 \mathrm{~mm}^{3}\right)$. In accordance with previous studies VOI locations were chosen in predefined cortical and subcortical gray and white matter. For each subject spectra from 12 different locations were acquired in two separate sessions.

\section{RESULTS AND DISCUSSION}

\section{Combination of Spectra}

For phased array coils with $n$ elements, the data needed for each quantified spectrum consists of $2 n+1$ time-domain signals (here $n=8$ yields 17): $S_{\mathrm{w}, \mathrm{b}}(t)$ denotes the single scan without water suppression acquired with the body coil in transmit-receive mode, $S_{\mathrm{w}, k}(t)$ refers to the set of $n$ single scans without water suppression obtained from the $n$ elements of the phased array coil after body coil excitation, and $S_{k}(t)$ represents the corresponding set of watersuppressed acquisitions. The situation is illustrated in Fig. 1, which depicts experimental data of a proton MRS study of human brain as recorded by the individual elements of an eight-channel phased array head coil. While the outer left column shows the single-scan spectra without water suppression, the second column contains the corresponding water-suppressed raw spectra. Because the phase behavior of the reference spectra is highly correlated to that of the water-suppressed spectra, the latter can be compensated for residual eddy current distortions by using the phase information $\phi_{\mathrm{w}, k}(t)$ of the former according to (15)

$$
S_{\mathrm{e}, k}(t)=S_{k}(t) \cdot e^{-i \phi_{\mathrm{w}, k}(t)} .
$$

The resulting spectra without eddy current effects are simultaneously corrected for zero-order phase differences and shown in the third column of Fig. 1. The subsequent summation procedure combines the individual spectra by using a weighted sum

$$
S_{\text {sum }}(t)=\sum_{k=1}^{8} w_{k} S_{\mathrm{e}, \mathrm{k}}(t),
$$

with weighting factors $w_{k}$ proportional to the SNR of the corresponding spectrum $(2,4)$. Here, the weighting factors were determined by

$$
w_{k}=A_{\mathrm{w}, \mathrm{k}} / \sum_{l=1}^{8} A_{\mathrm{w}, l},
$$

where $A_{\mathrm{w}, k}$ refers to the amplitude of the time-domain signal without water suppression (obtained by fitting an exponential decay to the data). For the example shown in Fig. 1 these weighting factors are given in the outer left column. They are in good agreement with the corresponding SNR values given in the third column of Fig. 1. Finally, the outer right column of Fig. 1 shows spectra and SNR values from a successive weighted summation of $N$ spectra ordered with respect to SNR,

$$
S_{\text {sum }, N}(t)=\sum_{k=1}^{N} w_{k} S_{\mathrm{e}, k}(t) .
$$

Both visual inspection and a quantitative analysis by LCModel indicate that summation of the four best spectra results in a steady improvement of the SNR, while contributions from the other coil elements have only a minor impact on the total signal quality. This behavior was typical for VOI locations in the outer cortex where a maximum of two to four coil elements provide a very strong signal. However, the situation is different in locations such as the thalamus, which are closer to the center of the brain and therefore further away from all elements of the circumscribing phased array head coil. Under these conditions all coil elements provide similar signal contributions to the net spectrum and the SNR of the summed spectrum increases monotonically with the number of spectra. In general, no case has been observed where the exclusion of the signal from a particular coil element resulted in an improvement of the final spectrum. On the other hand, because the SNR of the summed spectrum was never compromised by adding all spectra, this was chosen as a standard procedure for this work.

\section{Quantification of Absolute Metabolite Concentrations}

A prerequisite for the accuracy of the absolute reference method (with or without a phased array coil) is the quality 

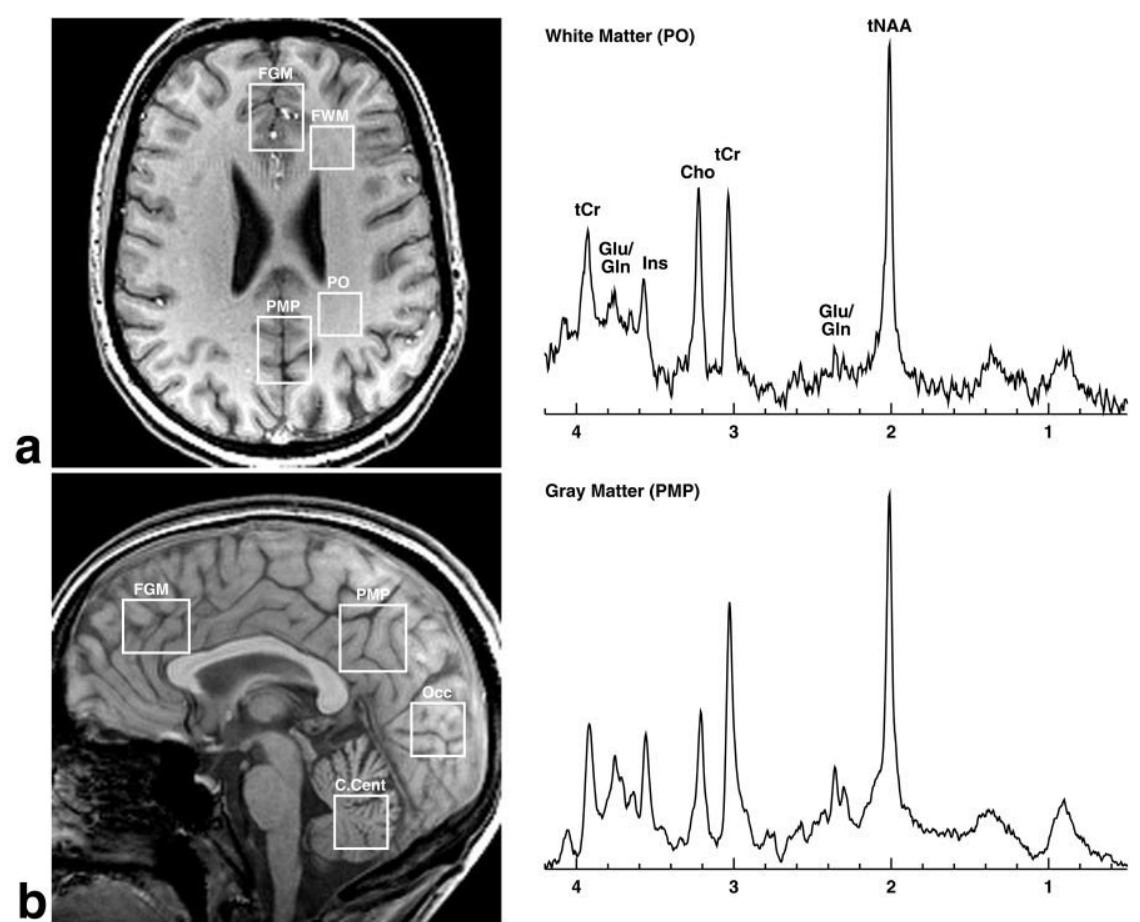

FIG. 3. (Left) VOI locations ( $T_{1}$-weighted 3D FLASH MRI) and (right) corresponding localized proton MR spectra (STEAM, TR/ $\mathrm{TE} / \mathrm{TM}=6000 / 20 / 10 \mathrm{msec}, 64$ accumulations) of a 21-year-old male subject acquired with an 8-channel phased array head coil after excitation by the body coil. (a) $\mathrm{PO}=$ parieto-occipital white matter (4.1 mL), (b) PMP = paramedian parietal gray matter $(12.5 \mathrm{~mL})$, (c) Tha = thalamus $(4.1 \mathrm{~mL}),(\mathrm{d}) \mathrm{C}$. Cent $=$ central part of cerebellum (8 $\mathrm{mL}$ ). For display purposes only, the spectra (scaled to arbitray units) were zero-filled (4096 complex data points), exponentially apodized (half width $221 \mathrm{msec}$, corresponding to $1 \mathrm{~Hz}$ line broadening), and manually phase corrected. FGM = frontal gray matter, FWM = frontal white matter, Occ = occipital gray matter, $\mathrm{C}$.Hemi $=$ cerebellar hemisphere.

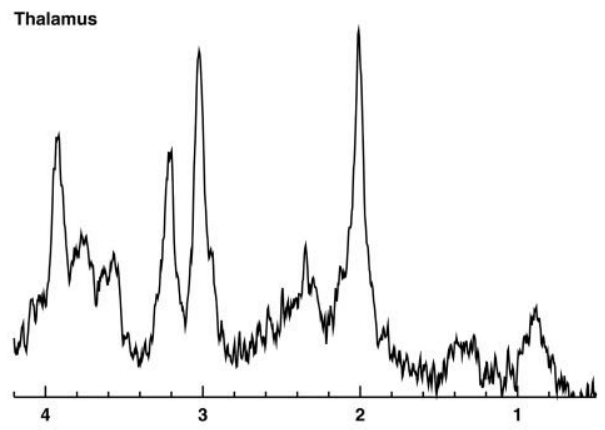

C
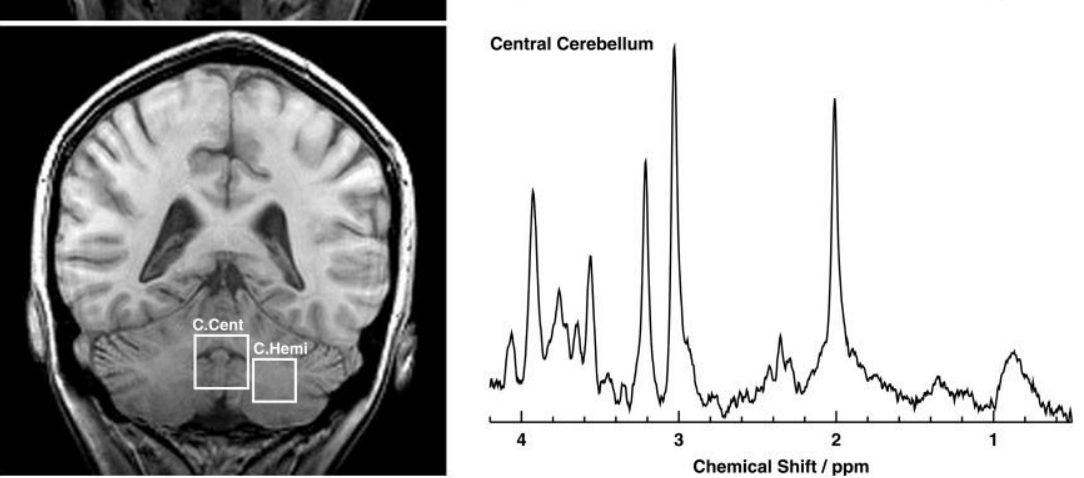

with which the transmitter amplitude of a reference pulse can be determined. In the present study, the manufacturer's automatic procedure employs a $90^{\circ}$ rectangular pulse, which is then used to calculate the power of other (shaped) pulses in a specific MRI or MRS sequence. The accuracy of this method was controlled by deliberately varying the nominal value calculated for the STEAM localization sequence and the body coil in transmit-receive mode. In fact in all cases, the maximum signal of a single-scan acquisition without water suppression was obtained for a flip angle (RF power), which was within $\pm 5 \%$ of the nominal value. The transmitter amplitude of the body coil $\mathrm{TA}_{\mathrm{bc}}$ which is given in volts by the manufacturer's software therefore adequately represents the actual loading and may be taken as a good measure for the inverse sensitivity.

When using a phased array coil in conjunction with body coil excitation as proposed here, then the product of $\mathrm{TA}_{\mathrm{bc}}$ with the ratio of the water signal reference amplitudes obtained with the body coil $A_{w, \text { bc }}$ and the (combined) phased array coil $A_{w \text {,sum }}$ corresponds to the inverse sensitivity of the phased array coil. Accordingly, the (virtual) transmitter amplitude of the phased array coil TA $\mathrm{T}_{\text {sum }}$ required for quantification of an absolute metabolite concentration yields 
Table 1

Metabolite Concentrations ( $\mathrm{mmol} /$ liter VOI $\pm \mathrm{SD}$ ) in Gray Matter and Thalamus

\begin{tabular}{|c|c|c|c|c|}
\hline & \multicolumn{3}{|c|}{ Gray matter } & \multirow{2}{*}{ Thalamus } \\
\hline & Frontal & Parietal & Occipital & \\
\hline tNAA & $7.2 \pm 0.7$ & $7.0 \pm 0.7$ & $7.2 \pm 0.8$ & $7.3 \pm 1.6$ \\
\hline NAA & $6.6 \pm 0.6$ & $6.5 \pm 0.7$ & $6.5 \pm 0.9$ & $6.4 \pm 1.3$ \\
\hline NAAG & $0.6 \pm 0.3$ & $0.4 \pm 0.3$ & $0.6 \pm 0.3$ & $0.9 \pm 1.1$ \\
\hline $\mathrm{tCr}$ & $5.9 \pm 0.6$ & $5.5 \pm 0.7$ & $4.9 \pm 0.6$ & $6.3 \pm 1.5$ \\
\hline Cho & $1.5 \pm 0.2$ & $1.0 \pm 0.1$ & $0.7 \pm 0.2$ & $1.7 \pm 0.5$ \\
\hline Ins & $3.7 \pm 0.4$ & $3.3 \pm 0.4$ & $2.9 \pm 0.4$ & $3.0 \pm 1.2$ \\
\hline Glu & $7.0 \pm 0.7$ & $6.4 \pm 0.8$ & $5.0 \pm 0.7$ & $4.7 \pm 2.4$ \\
\hline Gln & $2.9 \pm 0.7$ & $2.5 \pm 0.7$ & $2.2 \pm 0.6$ & $5.7 \pm 1.7$ \\
\hline VOI (mL) & 10.0 & 12.5 & 8.0 & 4.1 \\
\hline $\mathrm{FWHM}^{\mathrm{a}}(\mathrm{Hz})$ & $5.2 \pm 0.8$ & $5.8 \pm 0.8$ & $6.2 \pm 0.8$ & $9.5 \pm 1.7$ \\
\hline $\mathrm{SNR}^{\mathrm{a}}$ & $23 \pm 3$ & $25 \pm 4$ & $23 \pm 4$ & $5 \pm 1$ \\
\hline $\mathrm{SNR}_{\mathrm{VOI}}\left(\mathrm{mL}^{-1}\right)$ & $2.3 \pm 0.3$ & $2.0 \pm 0.3$ & $2.9 \pm 0.4$ & $1.3 \pm 0.2$ \\
\hline
\end{tabular}

Determined by LCModel.

$$
\mathrm{TA}_{\text {sum }}=\mathrm{TA}_{\mathrm{bc}} \cdot \frac{A_{\mathrm{w}, \mathrm{bc}}}{A_{\mathrm{w}, \mathrm{sum}}} .
$$

Finally, the following information was provided to LCModel for spectral analysis: (i) the weighted sum of the water-suppressed spectra from the different coil elements $S_{\text {sum }}(t)$ according to Eq. [2], (ii) the calculated transmitter reference amplitude $\mathrm{TA}_{\text {sum }}$, and (iii) a calibration factor which accounts for the fact, that the library of model spectra was acquired with a different coil.

\section{Phantom Experiments}

Using the aforementioned procedure, quantification of the $50 \mathrm{mM}$ creatine phantom yielded a creatine concentration of $48.9 \pm 2.3 \mathrm{mM}$. In more detail, the use of rather different coil loadings and VOI off-center positions resulted in a $\mathrm{TA}_{\mathrm{bc}}$ range of $275-322 \mathrm{~V}$, which translated into $\mathrm{TA}_{\text {sum }}$ values for the phased array coil of 223-459 V. Despite this large variation, the full range of evaluated creatine concentrations varied only moderately between 45.9 and $52.5 \mathrm{mM}$ with no obvious correlation to one of the reference ampli- tudes. Moreover, as demonstrated in Fig. 2, the accuracy of the absolute quantification revealed no systematic dependency on the position of the VOI within the accessible range of $\pm 50 \mathrm{~mm}$.

These results demonstrate that the proposed quantification method with combination of individual spectra from the different elements of a phased array coil provides a high accuracy for the determination of metabolite concentrations. The results are generally independent of both the loading of the used coils and the relative position within the phased array coil.

\section{Human Studies}

Representative proton MR spectra of human brain in vivo are shown in Fig. 3 together with images indicating the locations and dimensions of respective VOIs. In accordance with previous studies, VOIs were placed in gray matter of the paramedian frontal cortex $\left(20 \times 25 \times 20 \mathrm{~mm}^{3}\right.$ $=10 \mathrm{~mL})$, parietal cortex $\left(20 \times 25 \times 25 \mathrm{~mm}^{3}=12.5 \mathrm{~mL}\right)$, occipital cortex $\left(20 \times 20 \times 20 \mathrm{~mm}^{3}=8 \mathrm{~mL}\right)$, left and right thalamus $\left(16 \times 16 \times 16 \mathrm{~mm}^{3}=4.1 \mathrm{~mL}\right)$, and central

Table 2

Metabolite Concentrations ( $\mathrm{mmol} /$ liter VOI $\pm \mathrm{SD}$ ) in White Matter and Cerebellum

\begin{tabular}{|c|c|c|c|c|}
\hline & \multicolumn{2}{|c|}{ White matter } & \multicolumn{2}{|c|}{ Cerebellum } \\
\hline & Frontal & Parieto-occipital & Hemispheres & Central \\
\hline tNAA & $6.8 \pm 0.8$ & $7.6 \pm 0.8$ & $8.0 \pm 0.9$ & $7.1 \pm 1.1$ \\
\hline NAA & $5.9 \pm 0.7$ & $6.4 \pm 0.8$ & $6.1 \pm 0.8$ & $6.2 \pm 1.0$ \\
\hline NAAG & $0.9 \pm 0.5$ & $1.3 \pm 0.5$ & $1.9 \pm 0.8$ & $0.8 \pm 0.4$ \\
\hline $\mathrm{tCr}$ & $4.3 \pm 0.5$ & $4.3 \pm 0.5$ & $6.0 \pm 0.9$ & $7.5 \pm 1.0$ \\
\hline Cho & $1.8 \pm 0.2$ & $1.5 \pm 0.2$ & $1.9 \pm 0.2$ & $1.9 \pm 0.3$ \\
\hline Ins & $2.8 \pm 0.5$ & $2.8 \pm 0.5$ & $4.0 \pm 0.7$ & $4.3 \pm 0.7$ \\
\hline Glu & $3.9 \pm 1.1$ & $4.1 \pm 0.8$ & $5.0 \pm 1.4$ & $5.8 \pm 1.1$ \\
\hline Gln & $3.1 \pm 0.9$ & $2.2 \pm 0.7$ & $3.9 \pm 1.5$ & $3.8 \pm 1.4$ \\
\hline VOI (mL) & 4.1 & 4.1 & 4.1 & 8.0 \\
\hline $\mathrm{FWHM}^{\mathrm{a}}(\mathrm{Hz})$ & $6.5 \pm 1.0$ & $5.1 \pm 1.2$ & $7.2 \pm 1.2$ & $5.8 \pm 1.3$ \\
\hline $\mathrm{SNR}^{\mathrm{a}}$ & $9 \pm 2$ & $12 \pm 2$ & $7 \pm 1$ & $17 \pm 3$ \\
\hline $\mathrm{SNR}_{\mathrm{VOI}}\left(\mathrm{mL}^{-1}\right)$ & $2.1 \pm 0.4$ & $2.9 \pm 0.5$ & $1.7 \pm 0.2$ & $2.1 \pm 0.4$ \\
\hline
\end{tabular}


cerebellum $\left(20 \times 20 \times 20 \mathrm{~mm}^{3}=8 \mathrm{~mL}\right)$ as well as in white matter of the left and right frontal and parieto-occipital cortex and left- and right-hemispheric cerebellum (all $16 \times$ $16 \times 16 \mathrm{~mm}^{3}=4.1 \mathrm{~mL}$ ). Tables 1 and 2 summarize the concentrations of major cerebral metabolites as well as mean linewidths and SNR values.

It is noteworthy that the full width at half maximum (FWHM) is approximately 50\% larger than in studies performed at $2.0 \mathrm{~T}(16,17)$ and approximately $100 \%$ larger than at $1.5 \mathrm{~T}(18)$. This observation is primarily due to a more pronounced $T_{2}{ }^{*}$ decay at higher fields and only partly caused by a shortening of $T_{2}$ (19). The increased linewidth is in turn responsible for the fact that only a small portion of the higher SNR obtained here can be ascribed to the 3-T field strength. This is in agreement with previous observations reporting an SNR increase of less than $30 \%$ when going from 1.5 to $3.0 \mathrm{~T}(20)$. Thus, the improved SNR clearly stems from the use of a phased array coil. As indicated by the SNR per unit volume $\left(\mathrm{SNR}_{\mathrm{VOI}}\right)$ given in Tables 1 and 2, this notion is supported by the high values $\left(2.9 \mathrm{~mL}^{-1}\right)$ obtained for gray and white matter in occipital cortex, which is close to one or two coil elements as opposed to the slightly lower values $(2.0-2.3$ $\mathrm{mL}^{-1}$ ) for more distant VOI locations in frontal or parietal cortex. The much lower $\mathrm{SNR}_{\mathrm{VOI}}$ for the thalamus reflects both its central position and the poor linewidth achievable in this region, which most likely reflects both a small $T_{2}$ * value due to the enhanced iron content and motion induced by pulsatile flow from large vessels.

The concentrations of most cerebral metabolites in Tables 1 and 2 including total $N$-acetylaspartate (tNAA), creatine and phosphocreatine $(\mathrm{tCr})$, and choline-containing compounds (Cho) are in general agreement with previous findings in young adults as, for example, reported from our own lab using 2.0-T acquisitions with a volume coil (17). Nevertheless, in selected cases, that is for a particular metabolite and/or location, differences have to be acknowledged. For example, glutamate (Glu) and myoinositol (Ins) concentrations are about 20-30\% lower than in our previous study. At least for the strongly coupled Glu resonances a possible explanation stems from the fact that the metabolite quantification by LCModel is sensitive to low SNR. In fact, by adding Gaussian noise to a sample spectrum, it can be shown that the analysis of low-SNR spectra bears a tendency for overestimating Glu. This effect may have contributed to its higher level found before. Moreover, apart from somewhat reduced NAA and $\mathrm{N}$ acetylaspartylglutamate (NAAG) concentrations in white matter, thalamus, and cerebellum, the reported NAA gradient from frontal to occipital gray matter (16) could not be confirmed as all NAA and NAAG concentrations in gray matter were similar.

\section{CONCLUSIONS}

The method proposed here provides a simple and robust strategy for the quantification of absolute metabolite concentrations using MRS with phased array coils. The approach has little impact on the total measuring time as only a single scan without water suppression must be added to the equivalent approach for transmit-receive coils. As shown here for quantitative proton MRS of the human brain, the method takes full advantage of the improved SNR offered by phased array coils. Of course, this particularly applies to VOI locations in the vicinity of one or two of the individual coil elements. Finally, it should be emphasized that the calibration factor required for the phased array coil only depends on the properties of the body coil in transmit-only mode. Therefore, it is possible to apply the quantification method to different phased array and/or surface coils without the need for a recalibration.

\section{REFERENCES}

1. Wright SM, Wald LL. Theory and applications of array coils in MR spectroscopy. NMR Biomed 1997;10:394-410.

2. Wald LL, Moyher SE, Day MR, Nelson SJ, Vigneron DB. Proton spectroscopic imaging of the human brain using phased array detectors. Magn Reson Med 1995;34:440-445.

3. Prock T, Bassouls E, Collins DJ, Leach MO. SNR discrimination of useful MRS data from a phased array. In: Proceedings of the 8th Annual Meeting of ISMRM, Denver, 2000; p 8562.

4. Prock T, Collins DJ, Dzik-Jurasz ASK, Leach MO. An algorithm for the optimum combination of data from arbitrary magnetic resonance phased array probes. Phys Med Biol 2002;47:39-46.

5. Barker PB, Soher BJ, Blackband SJ, Chatham JC, Mathews VP, Bryan RN. Quantitation of proton NMR spectra of the human brain using tissue water as an internal concentration reference. NMR Biomed 1993; $6: 89-94$.

6. Soher BJ, Hurd RE, Silasuta N, Barker PB. Quantitation of automated singlevoxel proton MRS using cerebral water as an internal reference. Magn Reson Med 1996;36:335-339.

7. Ernst T, Kreis R, Ross BD. Absolute quantitation of water and metabolites in the human brain. I. compartments and water J Magn Reson B 1993;102:1-8.

8. Michaelis T, Merboldt KD, Bruhn H, Hänicke W, Frahm J. Absolute concentrations of metabolites in the adult human brain in vivo: quantification of localized proton MR spectra. Radiology 1993;187:219-227.

9. Hoult DI, Richards RE. The signal-to-noise ratio of the nuclear magnetic resonance experiment. J Magn Reson 1976;24:71-85.

10. van der Klink JJ. The NMR reciprocity theorem for arbitrary probe geometry. J Magn Reson 2001;148:147-154.

11. Danielsen ER, Henriksen O. Absolute quantitative proton NMR spectroscopy based on the amplitude of the local water suppression pulse. Quantification of brain water and metabolites. NMR Biomed 1994;7: 311-318.

12. Danielsen ER, Michaelis T, Ross BD. Three methods of calibration in quantitative proton MR spectroscopy. J Magn Reson B 1995;106:287291.

13. Ogg RJ, Kingsley PB, Taylor JS. WET, a $T_{1}$ - and $B_{1}$-insensitive watersuppression method for in vivo localized $1 \mathrm{H}$ NMR spectroscopy. J Magn Reson B 1994;104:1-10.

14. Provencher SW. Estimation of metabolite concentrations from localized in vivo proton NMR spectra. Magn Reson Med 1993;30:672-679.

15. Klose U. In vivo proton spectroscopy in presence of eddy currents. Magn Reson Med 1990;14:26-30.

16. Pouwels PJW, Frahm J. Regional metabolite concentration in human brain as determined by quantitative localized proton MRS. Magn Reson Med 1998;39:53-60.

17. Pouwels PJW, Brockmann K, Kruse B, Wilken B, Wick M, Hanefeld F, Frahm J. Regional age dependence of human brain metabolites from infancy to adulthood as detected by quantitative localized proton MRS. Pediatr Res 1999;46:474-485.

18. Helms G. Analysis of 1.5 Tesla proton MR spectra of human brain using LCModel and an imported basis set. Magn Reson Imaging 1999;17: 1211-1218.

19. Träber F, Block W, Lamerichs R, Gieseke J, Schild HH. ${ }^{1} \mathrm{H}$ metabolite relaxation times at 3.0 Tesla: measurements of $T_{1}$ and $T_{2}$ values in normal brain and determination of regional differences in transverse relaxation. J Magn Reson Imaging 2004;19:537-545.

20. Barker PB, Hearshem DO, Boska MD. Single-voxel proton MRS of the human brain at 1.5 T and 3.0 T. Magn Reson Med 2001;45:765-769. 